\title{
Der Südpazifik: Inselwelten zwischen verfassungsrechtlicher Modernität und gesellschaftlicher Tradition
}

\author{
Von Jörg Menzel
}

\section{A. Einleitung}

Die Südsee ist ein mythischer Raum. Der französische Seefahrer Louis Antoine de Bougainville fand hier (in Tahiti) die "edlen Wilden" Rousseaus, Jack London den Stoff für phantastische Geschichten, Paul Gauguin seine traurig-schönen Motive. Juristerei ist (für gewöhnlich) wenig mythisch und so bleibt der Verfassungsraum Südsee ${ }^{1}$ hierzulande selbst in der Zeitschrift, deren Geburtstag hier zu begehen ist, bislang fast unentdeckt ${ }^{2}$. Geographisch wie ethnisch teilt sich die Region in die "schwarzen", die "vielen" und die "kleinen" Inseln: Melanesien ${ }^{3}$ liegt im Südwesten, Polynesien ${ }^{4}$ im Südosten, Mikronesien ${ }^{5}$ im Norden. Die Übergänge zwischen den Ethnien, Kulturen und traditionellen Gesellschaftsstrukturen sind fließend ${ }^{6}$. Staatsrechtlich handelt es sich heute um die Region der Klein-, Kleinst- und Mikrostaaten, abhängiger Territorien und kolonialer Restbestände ${ }^{7}$. Die Breite

Zu den Begriffs- und Abgrenzungsfragen (Südpazifik, Südsee, Ozeanien) vgl. Werner Kreisel, Die pazifische Inselwelt, 1991, S. $1 \mathrm{ff}$.

2

Vgl. aber Peter G. Sack, »Law« and »Custom« in Papua New Guinea: Separation, Unification or Co-operation?, VRÜ 1987, 329 ff.; Jörg Menzel, Paradiesische Zustände? - Grundlagen und Entwicklungstendenzen von Verfassung und Recht in Fidschi, VRÜ 1987, S. 42 ff.

3 Papua-Neuguinea, Solomonen, Vanuatu, Fidschi, Neukaledonien.

Tonga, West-Samoa, Tuvalu, Neuseeland, Cook-Inseln, Niue, Tokelau, Amerikanisch-Samoa, Hawaii, Französisch-Polynesien, Wallis und Futuna, Osterinseln, Pitcairn.

5

Vereinigte Staaten Mikronesiens, Kiribati, Nauru, Palau, Marshall-Inseln, Nördliche Marianen, Guam.

6 Nach langem Streit geht man heute für die gesamte Region überwiegend von einer aus dem asiatischen Raum erfolgten Besiedlung aus. Thor Heyerdahls berühmte Kon-Tiki-Tour, mit der die Besiedelung Polynesiens von Südamerika aus belegt werden sollte, hat hieran nichts geändert. Ausführliche Zusammenfassung des Forschungsstandes zur Besiedelungsgeschichte bei Werner Kreisel (Anm. 1), S. 67 ff.

7 Insgesamt 14 Staaten und weitere 13 abhängige Territorien mit politischem Eigenleben hat kürzlich Guy Powles tabellarisch zusammengestellt (Transformation of Customary Law. Legal Pluralism in Pacific Islands States. Paper for Pacific Islands Legal Of ficers Meeting, Port Vila 1995, Anhang). Das aktuelle "Pacific Islands Yearbook", (17. Auflage, Suva (Fiji), 1994) berücksichtigt insgesamt 34 Einheiten (wobei die Teilstaaten der Vereinigten Staaten von Mikronesien und Palaus ausgeklammert sind). 
des Themas und der Variantenreichtum in der Region machen die Auswahl des zu Berichtenden schwer, aber unvermeidlich: Geleistet werden kann hier nicht mehr als ein kursorischer Überblick.

\section{B. Zur Rechts- und Verfassungsgeschichte}

Inhaltlich soll es im Folgenden vor allem - in der Begrifflichkeit Max Webers gesprochen um das Verhältnis von traditionaler und legaler Herrschaft gehen. Sinnvoll läßt sich dies nur vor dem gegebenen historischen Hintergrund besprechen, dem daher zunächst einige Hinweise gelten sollen.

\section{Das voreuropäische Zeitalter}

James Cook und seine entdeckenden Zeitgenossen stoßen in Polynesien (wie schon die Spanier zuvor in Mikronesien) zumeist auf hierarchiegewöhnte Aristokratien. Zentrale Institutionen, Anklänge staatlicher Organisation, sind zwar nicht immer und überall gleich wirksam (insgesamt scheint am "Vorabend" der europäischen Entdeckung vieles im Umbruch zu sein), aber jedenfalls als Idee virulent ${ }^{8}$. Tonga kennt wohl etwa seit etwa dem 10. Jahrhundert n. Chr. den sog. Tu'i Tonga, ein gemeinsames (zehntausende Menschen erfassendes) Oberhaupt, dessen Funktion sich aber im Laufe der Jahrhunderte auf den sakralen Bereich verlagert ${ }^{9}$. Starke zentrale Herrschaftsstrukturen bzw. die Erinnerung an sie lassen sich gesichert auch für Samoa, Tahiti und insbesondere Hawaii nachweisen.

Völlig anders ist die Situation allerdings im westlichen Melanesien, in den Gebieten des heutigen Papua-Neuguineas, der Solomonen, Neukaledoniens und Vanuatus. Hier stoßen die ersten Europäer auf einen definitiv vorstaatlichen Zustand. Kleine Einheiten von etwa 20 bis zu maximal einigen hundert Mitgliedern bilden zumeist die einzige Form sozialer Organisation. Die politischen Strukturen sind tendentiell egalitär, Führungsstrukturen, soweit überhaupt vorhanden, bleiben eher informell. Der melanesische "big man" (soweit es ihn gibt) erlangt seine Stellung aufgrund von Leistung und Anerkennung, anders als der

Wenn etwa Paul Laband (Staatsrecht des Deutschen Reiches, 5. Aufl., Bd. 2, 1911, S. 272 ff.) offensichtlich ausgehend von den Zuständen in Melanesien - für die Südsee einen durchweg "wilden", vorrechtlichen Zustand annimmt, beruht dies schlicht auf mangelnder Differenzierung.

9 Vgl. I.C. Campbell, Island Kingdom. Tonga ancient \& modern, Christchurch 1992, S. 7; Sione Latukefu, The Tongan Constitution. A brief history to celebrate its Centenary, Nuku'alofa (Tonga) 1975, S. 1. Alle Schätzungen sind insoweit freilich recht unsicher. 
hereditär legitimierte polynesische Chief ${ }^{10}$. Im Hinblick auf solche Gegenüberstellungen ist - bei aller Richtigkeit in der Grundaussage - in zweierlei Hinsicht einige Vorsicht gebo ${ }^{-}$ $\operatorname{ten}^{11}$ :

Inzwischen ist erstens völlig klar, daß weder die Vererbung von Führungspositionen in Melanesien gänzlich unbekannt, noch die persönliche Überzeugungskraft für die Herrscherauslese in Polynesien bedeutungslos ist. Der melanesische big man läßt sich dem polynesischen chief dementsprechend nur idealtypisch gegenüberstellen. Gerade im geographischen Grenzbereich sind die Trennlinien unscharf: Fidschi im Osten Melanesiens etwa weist traditionell hierarchisch-hereditäre und damit typisch polynesische Ordnungsstrukturen auf.

Zweitens wurde gerade in jüngster Zeit anhand einer Reihe von Beispielen aufgezeigt, daß vieles von dem, was im Südpazifik heute als "customary" oder "traditional" bezeichnet wird, sich erst nach dem Kontakt mit Europäern (und oft gerade in seiner Folge) entwickelt hat $^{12}$; was als Tradition bezeichnet (man könnte gelegentlich auch sagen: verkauft) wird, ist dann oft kaum mehr als eine Erfindung im Kontext moderner politischer Auseinandersetzungen $^{13}$. Die Berufung auf Herkommen gehört eben zu den schneidigsten Schwertern im politischen Streit der Region.

Über das "Recht" der südpazifischen Bevölkerungen der voreuropäischen Zeit sind die Kenntnisse regional sehr unterschiedlich, insgesamt aber recht unsicher ${ }^{14}$. Durchweg handelt es sich um Gesellschaften mit starker Gruppenbezogenheit. Dies bedeutet nicht, daß man in einem auf Freiwilligkeit beruhenden, vorrechtlichen Naturzustand lebte. Die Regelhaftigkeit des Gebens und Nehmens, die "Reziprozität", haben Rechtsethnologen wie

Grundlegend wohl M. Sahlins, Poor Man, Rich Man, Big Man, Chief: Political Types in Melanesia and Polynesia, in: Comparative Studies in Society and History 5 (1962-1963), 285 ff.

11

Relativierend gegenüber Sahlins insb. B. Douglas, Rank, Power, Authority: A Reassessment of Traditional Leadership in South Pacific Societies, in: Journal of Pacific History 14 (1979), S. 22 ff.; vgl. auch Peter Larmour, States and Societies in the Pacific Islands, Pacific Studies, Vol. 15 (1992), 99 (105).

Vgl. für Santa Isabel (Solomonen) Geoffrey M. White, The Discourse of Chiefs: Notes on a Melanesian Society, in: The Contemporary Pacific 1992, S. $73 \mathrm{ff}$.

Eindrucksvoll hierzu Roger M. Keesing, Creating the Past: Custom and Identity in the Contemporary Pacific, in: The Contemporary Pacific, Spring/Fall 1989, S. 19 ff. Kritisch auch Stephanie Lawson, The Politics of Tradition: Problems for political legitimacy an democracy in the South Pacific, in: Pacific Studies 16 (1993), No. 2 (June). chen auf die wenigen archäologischen Funde, mündliche Überlieferungen sowie auf die Reiseberichte früher Entdecker, Abententeurer und Missionare, deren Zuverlässigkeit naturgemäß erheblich variiert. 
Richard Thurnwald und Bronislaw Malinowski gerade im Südpazifik erkannt ${ }^{15}$. Auch findet sich - jedenfalls im Südpazifik - die durchaus drakonische Sanktionierung devianten Verhaltens ${ }^{16}$. Dies gilt nicht nur unter den schwierigen Lebensbedingungen des westlichen Melanesiens, sondern auch und gerade in den natürlichen "Überflußgesellschaften" des östlichen Polynesiens. Als Beleg für die Vorstellung vom glückselig-urkommunistischen Naturzustand (noble savage) eignen sich weder die ausgef eilt aristokratischen Gebiete Polynesiens und Mikronesiens noch die intertribal kämpfenden Gruppen Melanesiens.

\section{Das europäisch-koloniale Zeitalter}

In den meisten ${ }^{17}$ Gebieten des Südpazifiks setzt der Europäer-Kontakt in der zweiten Hälfte des 18. Jahrhunderts ein. Er führt zu schnellen und umfassenden Umwälzungen. In Polynesien ist es zunächst vor allem die konsequente Christianisierung, die das Leben und die gesellschaftlichen Regeln grundlegend verändert. Politisch forcieren europäischer Einfluß (und Waffentechnik) großräumliches Denken. Die Zeit zwischen dem ersten EuropäerKontakt und der Kolonialisierung ist nicht nur in Fidschi und West-Samoa ${ }^{18}$ beherrscht von kriegerischen Auseinandersetzungen. In Melanesien schreitet zwar die Christianisierung zunächst schleppender voran. Aber auch hier ist der gesellschaftliche Wandel, den der Kontakt mit Sandelholz-, Menschen-, Waffen- und sonstigen Händlern mit sich bringt, grundsätzlich, und auch hier befördert europäischer Einfluß die Idee großflächiger politischer Organisation. Nach und nach ist auch in Melanesien verbreitet von chiefs und gar von "paramount chiefs" die Rede. Es ist nicht zuletzt die Zeit der Erfindung neuer Traditionen ${ }^{19}$.

15

Ausführlich Marcel Mauss, Die Gabe. Form und Funktion des Austauschs in archaischen Gesellschaften, Frankfurt a.M. 1994. (franz. Originalausg. 1950).

16

Für die deutschen Südpazifik-Gebiete nach wie vor wertvoll die Berichte von Hermann Trimborn (Mikronesien), Richard Thurnwald (Melanesien) und Erich Schultz-Ewerth (West-Samoa) in: Erich Schultz-Ewerth / Leonhard Adam (Hg.), Das Eingeborenenrecht, 2. Bd., Stuttgart 1930, S. 435 ff.; jüngst etwa Hermann Joseph Hiery, Das Deutsche Reich in der Südsee (1900 - 1921). Eine Annäherung an die Erfahrungen verschiedener Kulturen, Göttingen 1995, S. 96 ff. m.w.N.

Im westlichen Hochland Papua-Neuguineas etwa ist dies nicht vor den 30er Jahren des 20. Jhdts.; vgl. Peter Larmour (Anm. 11), S. 104. Der Bereich der nördlichen Marianen und Guams geraten umgekehrt schon im frühen 16. Jahrhundert in (gewaltgeprägten) Kontakt mit den Spaniern; vgl. hierzu näher Gerd Hardach, König Kopra. Die Marianen unter deutscher Herrschaft 1899-1914, 1990, S. $18 \mathrm{ff}$.

Die klassische Beschreibung stammt aus der Feder von Robert Louis Stevenson, A footnote to history. Eight Years of Trouble in Samoa (z.B. in: The Works of Robert Louis Stevenson, Vol. XVII, Swanston ed., London o.J., S. 5 ff.).

Instruktiv a m Beispiel Santa Isabels (Solomonen) White (Anm. 12), passim. 
Formal findet europäisches Recht spätestens mit der Kolonialisierung Eingang in den Südpazifik; hiermit korrespondiert die Zurückdrängung traditioneller Rechtsstrukturen. Frankreich setzt, wie in den meisten seiner Kolonialgebiete, so auch in Französisch-Polynesien konsequent auf Assimilierung und läßt traditionelles Recht sowie vorgefundene Herrschaftsstrukturen konsequent unberücksichtigt. Etwas anders stellt sich die Situation in Neukaledonien dar, wo die Assimilierung an dem Widerstand der indigenen Bevölkerung scheitert und schon Ende des 19. Jahrhunderts einer partiellen Einräumung von Selbstverwaltungsrechten zugunsten der (inzwischen in Reservatgebiete verdrängten) Ureinwohner weicht ${ }^{20}$. Fidschi gilt als Musterbeispiel der englischen Politik des "indirect rule". Der Status der einheimischen Chiefs bleibt hier im Grundsatz unangetastet, sie werden in die Verwaltung einbezogen und behalten Entscheidungsmacht auf ihren jeweiligen Ebenen. Spezielle Fijian Courts sind für die fidschianischen Angelegenheiten und damit vor allem auch für solche des Brauchtums zuständig, die indigene Elite der Chiefs erhält mit ihrem "Great Council" schon bald ein zentrales Gremium. Das englische Modell bedingt den vergleichsweise weiterreichenden Erhalt autochtoner Strukturen, wobei Einwirkungen auf dieselben nicht ausbleiben (traditionelle Eliten werden limitiert und gleichzeitig stabilisiert; Brauchtum wird kodifiziert und damit unweigerlich modifiziert). Ganz eigenartig gestaltet sich die Kolonialzeit in den Neuen Hebriden (heute Vanuatu), die seit 1907 kondominial von England und Frankreich beherrscht werden. Dort bildet sich nebeneinander ein französisches und ein britisches Verwaltungs- und Rechtssystem aus. Es gibt zwei Systeme der Erziehung, Polizei, Krankenversorgung, zwei Hymnen, zwei Währungen, drei offizielle Sprachen ${ }^{21}$. Der Rechtskreise sind gar vier: neben das französische und das englische Recht tritt das gemeinsam kondominiale sowie das indigene Gewohnheitsrecht. Das Ergebnis ist, wie ein Beobachter schreibt, ein "einzigartiges Experiment in Rechtspluralismus" ${ }^{22}$. Das für die indigene Bevölkerung geltende Recht bleibt in vielem unklar. Einzelne Gesetze - etwa ein Native Criminal Code ${ }^{23}$ - werden kondominial erlassen, andere Bereiche bleiben ungeklärt. Insgesamt scheint das kondominiale Experiment wenig erfolgreich: In Anlehnung an John Miltons "Paradise Lost" ist landläufig von der Regierungsform des "Pandemoniums" die Rede.

Auch das Deutsche Reich ist für einige Jahre Kolonialmacht im Südpazifik. Es bedient sich sowohl in West-Samoa als auch in Mikronesien vorgefunder Ordnungsstrukturen ${ }^{24}$. In

20 21

22

23

24

Hier Eric Agostini, Aboriginal Rights in New Caledonia, in: Law and Anthropology, Vol. 2, 1989.

Vgl. David Weisbrot, Custom, Pluralism, and Realism in Vanuatu: Legal Development and the Role of Customary Law, in: Pacific Studies 13 (1989), S. 65 ff.

Weisbrot, (Anm. 21).

Joint Regulation No. 6 of 1927; vgl. Weisbrot (Anm. 21), S. 68.

Vgl. hierzu (wenn auch juristisch etwas unpräzise) Hiery' (Anm. 16), S. 105 ff. 
West-Samoa (der "Perle der Südsee" ${ }^{\prime 25}$ ) werden die aristokratische Gesellschaftsordnung jedenfalls im Grundsatz geachtet und die besonders kraftvollen dörflichen Entscheidungsgewalten auf dem Verordnungsweg sogar ausdrücklich anerkannt ${ }^{26}$. Einwirkungen bleiben auch hier nicht aus: Politisch aufsässige Mitglieder des Adels werden bei Bedarf deportiert, das Sanktionsmittel der Verbannung aus dem Dorf wird untersagt, mit der Einrichtung der Land- und Titelkommission wird der Adelsstand (auf Dauer) "verrechtlicht" und damit ebenfalls in das gewohnte Gefüge eingegriffen. Zu einem auch im Reichstag diskutierten Rechtsskandal kommt es 1905 auf den Marshall-Inseln, wo Landeshauptmann Brandeis Verstöße gegen das traditionelle Standeswesen - für die nach traditionellem Recht der Tod verhängt worden wäre - abgeschwächt mit der Prügelstrafe ahnden läßt, ein nach der Strafverordnung für Eingeborene aus dem Jahr 1890 ausdrücklich untersagtes Mittel ${ }^{27}$. Bemerkenswert ist auch die deutsche Verwaltungsorganisation in Neuguinea. Während die übrigen Kolonialmächte in West-Melanesien die "indirect rule" wegen fehlender indigener Ordnungsstrukturen für ausgeschlossen halten, baut der zweite deutsche Gouverneur Hahl die bescheidenen Ansätze hierzu zielgerichtet aus und instrumentalisiert sie als Träger der Ordnungsgewalt ${ }^{28}$.

Ein Sonderfall ist das Königreich Tonga; Tonga wird nicht Kolonie, sondern (nachdem Deutschland seine Interessen im Tausch gegen diejenigen in West-Samoa aufgegeben hat) lediglich englisches "Protektorat". Die Europäisierung des Rechts findet freilich auch hier statt, in mancher Hinsicht erfolgt sie sogar konsequenter als in den Nachbargebieten. Bereits seit 1839 wird in Tonga Recht kodifiziert ${ }^{29}$. Der König formalisiert hiermit schrittweise die Entmachtung der traditionellen Adelsschicht und festigt damit seine eigene Position, die ihn beratenden Missionare, die die Rechtstexte regelmäßig verfassen, setzen christliches Gedankengut durch. Dörfliche Selbstverwaltung nach überlieferten Regeln, wie sie etwas in West-Samoa und Fidschi unter kolonialer Macht überlebt, wird in Tonga schon im 19. Jahrhundert durch ein zentrales, auf das Königshaus zugeschnittenes Verwaltungssystem ersetzt.

So ausdrücklich Gouverneur Wilhelm Solf in der Reichstagsdebatte vom 6. März 1902, zitiert nach Hiery' (Anm. 16), S. 29. Verordnung betr. die Selbstverwaltung der Samoaner vom 27.01. u. 16.2.1901. Vgl. die Darstellung bei Hiery' (Anm. 16), S. $107 \mathrm{ff}$.

Vgl. Hiery' (Anm. 16), S. 115 f.; Horst Gründer, Geschichte der deutschen Kolonien, 3. Auflage, 1995, S. $171 \mathrm{f}$.

Übersicht über die frühen Law Codes des 19. Jhdts. bei Campbell (Anm. 9), S. 238 ff.; näher Latukefu (Anm. 9), S. $14 \mathrm{ff}$. 


\section{Das postkoloniale Zeitalter}

West-Samoas Schritt in die Unabhängigkeit eröffnet im Jahr 1962 die Dekolonialisierung des Südpazifiks ${ }^{30}$. England, Australien, Neuseeland und die USA entlassen die meisten ihrer Kolonien, Schutz- und Treuhandgebiete schrittweise in die Unabhängigkeit oder räumen ihnen einen Status weitgehender Selbständigkeit ein. Frankreich kann jedenfalls den Schritt des kondominal mit England verwalteten Vanuatus (der vormaligen Neuen Hebriden) in die Unabhängigkeit nicht verhindern.

Von einem nacheuropäischen Rechtszeitalter im Südpazifik kann aber nicht ernsthaft gesprochen werden. Formaljuristisch hat die Region ihr Fundament nach wie vor im angloamerikanischen Rechtskreis (in Vanuatu steht neben dem englischen formal auch das französische Recht in Geltung, faktisch ist letzteres aber im Verschwinden begriffen ${ }^{31}$ ). Der Schritt in die Unabhängigkeit hat zwar Modifikationen mit sich gebracht (vor allem eben die Einführung geschriebener Verfassungen), im wesentlichen ist die Entwicklung aber durch Kontinuität gekennzeichnet ${ }^{32}$. Eine andere Frage ist, in welchem Umfang förmlichem Recht in den entlegeneren Gebieten überhaupt eine prägende Rolle zuwächst. Daß alltägliche Konflikte auf äußeren Inseln und in entlegenen Dörfern mangels staatlichen Zugriffs bis heute zumeist auf der Grundlage lokaler Traditionen bereinigt werden, ist für viele der südpazifischen Staaten evident und unbestritten ${ }^{33}$. Dies ändert indes nichts an der Struktur des formalen staatlichen Rechts und seiner Zugehörigkeit zum Rechtskreis des Common Law. Das sei vorweggeschickt, bevor nun die Aufmerksamkeit den speziellen Elementen eines "pacific way" in Staat, Verfassung und Recht der Region gilt.

Es folgen Nauru (1968), Fidschi (1970), Papua-Neuguinea (1975), Tuvalu und die Solomonen (1978) und Kiribati (1979).

31 Zu den bislang erfolglosen Versuchen, die Gesetze Vanuatus in französischer Sprache zu publizieren, ausführlich der "Second Special Report on the Observance of Multilingualism" der (frankophonen) Ombudsfrau (1996), S. 12.

Praktisch alle in der Region tätigen Juristen haben ihre Ausbildung an englischen, australischen, neuseeländischen oder amerikanischen Universitäten erhalten, ebenso wie der überwiegende Anteil des Lehrpersonals an der neu eingerichteten juristischen Fakultät der Universität des Südpazifiks in Port Vila (Vanuatu). Die Beratungshilfe im Bereich der Gesetzgebung sowie die Berufung insb. neuseeländischer und australischer Richter zu den nur sporadisch tagenden höchsten Gerichten leisten ihr übriges, die fortdauernde Einbettung in den Common-Law-Rechtskreis zu gewährleisten.

33 In diesem Sinne scheint auch das von Neuseeland abhängige - derzeit um den Status der free association ringende - Tokelau im wesentlichen auf traditioneller Grundlage sein Recht zu finden. Vgl. A.H. Angelo, The Indiginous People of Tokelau and the Legal System, in: Law and Anthropology, Vol. 2, 1987; ders., "Tokelau", in: Michael A. Ntumy (ed.), South Pacific Islands Legal Systems, Honolulu 1993, S. 299. 


\section{Staatlichkeit}

Was zunächst die Frage der Staatlichkeit anbelangt, ist es weniger die Kleinheit, die für Probleme sorgt. Zwar ging Herbert Krüger in seiner Staatslehre noch davon aus, daß "der Kleinstaat die entscheidenden Merkmale und den Geist der Staatlichkeit nicht oder jedenfalls nicht vollständig" ausbilden könne ${ }^{34}$. Völkerrechtlich ist aber heute selbst MikroStaaten wie Tuvalu, Nauru oder Palau das Staatlichkeitstestat nicht wegen der Kleinheit zu verweigern $^{35}$, von den "größeren" wie Fidschi, den Solomonen, West-Samoa und Vanu$\mathrm{atu}^{36}$ ganz zu schweigen. Schwierig ist hier vielmehr - darauf sei aber nur hingewiesen die Qualifikation derjenigen Gebiete, die sich in einer "free association" befinden. Betroffen sind die Cook-Inseln und Niue (assoziiert mit Neuseeland) sowie die Vereinigten Staaten Mikronesiens, die Marshall-Inseln und Palau (assoziiert mit den USA). Für die Territorien selbst bringt ihre eigenartige völkerrechtliche Stellung durchaus Vorteile. So genießen etwa die Cook-Insulaner einerseits die volle neuseeländische Staatsbürgerschaft sowie bislang umfängliche neuseeländische Budgethilfe, andererseits haben sie sich (insoweit als Staat behandelt) auch Zugang zu den Quellen der internationalen Entwicklungshilfeverwaltung verschafft. Der Status der Cook-Inseln ist dabei juristisch autonom gewählt; die "Assoziation" kann zudem auch für die Zukunft einseitig aufgekündigt werden (ökonomische Vernunft wird solches freilich auf absehbare Zeit verhindern).

Wer zum Staatsvolk gehört und wer nicht, ist im Südpazifik gelegentlich schwer zu beantworten. Die Staatsbürgerschaft ${ }^{37}$ garantiert zwar üblicherweise das Recht zum Aufenthalt, aber durchaus noch keinen Status der "Meistbegünstigung". Bestimmte Rechte und Funktionen bleiben immer wieder den "indigenen" Einwohnern vorbehalten, so z.B. in Vanuatu das Präsidentenamt oder in West-Samoa die Möglichkeit zum Erwerb des so wichtigen Adels-Titels. Weitverbreitet ist die Benachteiligung oder der Ausschluß nicht indigener Staatsangehöriger beim Zugang zum Landeigentum ${ }^{38}$. Herausragende Bedeutung hat die Binnendifferenzierung der Staatsbürger in Fidschi. Die Nachfahren der von der englischen Kolonialverwaltung in das Land gebrachten indischen Kontraktarbeiter stellen heute - als Staatsbürger Fidschis - über $40 \%$ der Bevölkerung, zeitweilig waren sie gegenüber den ethnischen Fidschianern in der Mehrheit. Das Verhältnis der Bevölkerungsgruppen ist

34

35

36

37

38

Allgemeine Staatslehre, 1964, S. 5.

Vgl. nur Josef Isensee, Art.: "Staat", in: Staatslexikon der Görres-Gesellschaft, 7. Auflage, Bd. 5 (1989), Sp. 135.

Den Nrn. 150, 160, 168 und 169 in der Bevölkerungsrangliste der UN-Mitglieder, vgl. Vereinte Nationen 1997, S. 48.

Das Normenmaterial ist dokumentiert bei Hellmuth Hecker, Das Staatsangehörigkeitsrecht von Australien und Ozeanien, Frankfurt a.M 1980.

Hierzu etwa Ron Crocombe, Trends and Issues in Pacific Land Tenure, in: ders./Malama Maleisea (ed.), Land Issues in the Pacific, Christchurch 1994, S. 12. 
traditionell distanzgeprägt, eine Vermischung hat bislang praktisch nicht stattgefunden. Als aus den Wahlen des Jahres 1987 eine "indisch" dominierte Regierung hervorging (der Ministerpräsident war indigener Fidschianer), bildete dies 1987 den Anlaß zu zwei Militärcoups (den ersten des Südpazifiks). Die 1990 in Kraft gesetzte - demokratisch nicht legitimierte - Verfassung unterscheidet nun konsequent zwischen "Fijians", "Indians" und "others", mit Konsequenzen etwa im Wahlrecht, Landeigentumsrecht, Stipendien- und Subventionsrecht usw.

Das Staatsgebiet ist angesichts der Inselstruktur regelmäßig einfach bestimmt. Spannend ist die Frage der internen Verfügungsgewalt über das Land (und auch das es umgebende Meer). Sie ist das juristische Thema im Südpazifik. Dies gilt nicht nur dort, wo Land knapp und damit existenzieller Konfliktstoff ist (also in Polynesien und Mikronesien), sondern auch in den landreicheren Gebieten Melanesiens. Hier ist die Domäne traditionellen Rechts. Darauf wird noch zurückzukommen sein.

Das Problem der Staatsgewalt und damit zusammenhängend des Gewaltmonopols schließlich stellt sich zunächst vor allem in den westmelanesischen Staaten der Region. Vor der Ankunft der Europäer war die Vorstellung eines geeinten Herrschaftsgebietes hier unbekannt - und noch heute stößt sie auf Schwierigkeiten. Geht man von dem gängigen Verständnis aus, daß Staat nur ist, wer sein Gebiet zum überwiegenden Teil faktisch be ${ }^{-}$ herrscht ${ }^{39}$, gerät man insbesondere im Hinblick auf Papua-Neuguinea und wohl auch bei den Solomonen auf Schwierigkeiten. Hochland- und Buschregionen bleiben hier zum Teil konsequent unverwaltet. Sie sind noch heute Mekka anthropologischer Erforschung authentischer Gesellschaften. Das Parlament Papua Neuguineas sah sich noch 1977 genötigt, einen "Inter-Group Fighting Act" zu erlassen ${ }^{40}$, um dem Hobbes'schen Naturzustand in seinem Hinterland zu begegnen ${ }^{41}$ (der Supreme Court erklärte die in dem Gesetz enthaltene Pflicht des gruppenzugehörigen Einzelnen, seine Nichtteilnahme an solchen Kämpfen zu beweisen, wegen Verstoßes gegen die verfassungsrechtliche Unschuldsvermutung für nichtig ${ }^{42}$ ). Die Staaten dieser Region sehen sich auch immer wieder mit gewalttätigem Widerstand gegen den zentralen Herrschaftsanspruch konfrontiert. In Vanuatu erreichte dieser Widerstand im Rahmen des Übergangs zur Unabhängigkeit seinen Höhepunkt,

Zu ihm David Weisbrot, in: ders. (ed.), Law and Social Change in Papua New Guinea, Sydney et al 1982, S. $70 \mathrm{ff}$.

41 Eingehend Peter Hanser, Krieg und Recht. Wesen und Ursachen kollektiver Gewaltanwendung in den Stammesgesellschaften Neuguineas, Berlin 1985.

42 Vgl. Peter Bayne, Judicial Review and Pacific Constitutions, in: Pacific Perspective, Vol. 13, S. 2. 
Papua-Neuguinea steht aktuell vor dem ungelösten Problem des blutigen Konfliktes in Bougainville ${ }^{43}$.

Die (hierarchiegewöhnten) Gebiete Polynesiens und Mikronesiens sowie Fidschi sind in stärkerem Maße von staatlicher Autorität durchdrungen, faktische Schranken ergeben sich hier in erster Linie aus der Abgelegenheit einzelner Inseln. Ein Problem anderer Art stellt sich hier allerdings in West-Samoa, wo die staatliche Autorität in einem latenten Konflikt mit der traditionellen Autorität der dörflichen Entscheidungsträger - eben der matai - steht. Deren Machtposition in der samoanischen Gesellschaft ist bis heute so ausgeprägt, daß sie der formalen Staatsgewalt faktische Grenzen setzt. So bleiben etwa gerichtlich festgestellte Schadensersatzansprüche gegen dörfliche Entscheidungsträger mangels Durchsetzbarkeit gelegentlich wertlos ${ }^{44} .1993$ ordneten lokale Autoritäten des Dorfes Loma gar die Tötung eines gegenüber ihren Entscheidungen aufsässigen Mitbewohners an; zwei gehorsamspflichtige junge Dorfbewohner "vollstreckten" die Entscheidung ${ }^{45}$.

\section{Verfassungsstaatlichkeit}

In wohl keinem Gebiet der Welt finden sich heute so viele Verfassungen für so wenige Menschen ${ }^{46}$. Nicht nur die 14 unabhängigen bzw. frei assoziierten Staaten haben durchweg geschriebene Verfassungen, sondern auch abhängige Gebiete wie etwa Niue oder Amerikanisch-Samoa und selbstverständlich der US-Bundesstaat Hawaii. Hinzu kommen vier Gliedstaatenverfassungen in den Vereinigten Staaten von Mikronesien sowie 16 derselben in Palau (letzteres bei einer Gesamtbevölkerung von 17.000 Einwohnern). In der gesamten Region steht nur noch Neuseeland in der englischen Tradition der Nichtkodifikation. Die

Hierzu Tarcisius Kabutaulaka, Cohesion and Disorder in Melanesia: The Bougainville Conflict \& the Melanesian Way, in: New Politics in the Pacific, S. $63 \mathrm{ff}$.

Der berühmte Fall des Tariu Tavaiti vs. the Matai of Matautu ist geschildert bei Malama Maleisea, The Making of modern Samoa, Suva 1987, S. 214. Tavaiti war wegen diverser Maßnahmen des village fono (unter anderem einer Boykottanordnung gegenüber seinen Bussen) gerichtlich Schadensersatz zugesprochen worden, der nicht vollstreckt werden konnte.

Zum "Lona-Village"-Fall siehe Stephanie Lawson, Tradition versus Democracy in the South Pacific, 1996, S. 155 ff. Lawsons Darstellung sei nachgetragen, daß es - nach den nicht verifizierten Informationen des Verf. - immerhin zur Verurteilung und Inhaftierung der Täter gekommen sein soll.

Die Verfassungstexte der Region sind zusammengestellt in der Textsammlung "Pacific Constitutions", herausgegeben von der University of the South Pacific, Suva (Fiji) 1991, Vol. 1 Polynesia, Vol. 2 Independent States of Melanesia and Micronesia; Aktualisierungen (Std. Dezember 1996, aber unvollständig) in; Supplementary Readings, Port Vila (Vanuatu) 1997. Im übrigen sei für die Verfassungstexte verwiesen auf Albert P. Blaustein / Gisbert H. Flanz, Constitutions of the Countries of the World, sowie Constitutions of the Dependencies and special sovereignities, New York et al. 
Verfassungen selbst unterscheiden sich schon stilistisch erheblich, der ausladenden Verfassung Papua-Neuguineas (nach der indischen zur Zeit wohl die längste der Welt), steht das nüchtern-knappe Grundstatut Vanuatus gegenüber. Auffällig ist aber, daß die Verfassung regelmäßig wichtig ist; jedenfalls in den größeren Staaten (nach den regionalen Maßstäben etwa beginnend mit Tonga) spielt sie in Rechtsprechung wie politischem Diskurs eine maßgebliche und nicht zu unterschätzende Rolle.

\section{Staatsorganisation}

Staatsorganisationsrechtlich folgen die melanesischen Staaten im wesentlichen (mit zahlreichen Abweichungen en detail) dem englischen Westminster-Modell. In Mikronesien findet auch das amerikanische Modell der Präsidialverfassung einige Nachahmung. Eine interessante Mischung bietet Kiribati, wo der Präsident aus einer Vorschlagsliste des Parlaments vom Volk direkt gewählt wird, aber vom Vertrauen des Parlaments abhängig bleibt $^{47}$. Besonders bunt ist das Bild in Polynesien. Auch hier dominiert die Grundstruktur des Westminster-Modells, es finden sich aber zahlreiche Spezifika. West-Samoa gibt sich ein dezidert aristokratisches Wahlrecht, Tonga bleibt einstweilen (wie Otto Mayer vielleicht gesagt hätte) vollsäftiges Königtum ${ }^{48}$. Traditionelle Eliten verfügen aber nicht nur hier über eine verfassungsrechtlich herausgehobene Stellung. Versammlungen von Chiefs auf nationaler Ebene haben teils beratenden Einfluß (so in Vanuatu, auf den Cook- und den Marshall-Inseln), teils echte Entscheidungsbefugnisse (so in Fidschi oder in Yap (einem Teilstaat Mikronesiens).

Ob es sich bei der parlamentarischen Demokratie englischer Prägung um die für Melanesien ideale Regierungsform handelt, wird inzwischen vielfach bezweifelt, die Stärkung der Funktion des Staatsoberhauptes ${ }^{49}$ oder auch konkordanzdemokratische Modelle ${ }^{50}$ werden als Alternativen diskutiert ${ }^{51}$. Unübersehbar ist jedenfalls, daß Papua-Neuguinea, die Solomonen und auch Vanuatu seit Jahren unter erheblichen Funktionsstörungen in ihren politischen Systemen leiden. Schon die Parteiensysteme sind instabil, dementsprechend sind es auch die parlamentarischen Mehrheiten und Regierungen. Das Geflecht persönlicher

47

48

Constitution, Section $30 \mathrm{ff}$.

8 Zur Auseinanderentwicklung dieser Systeme, die vor 200 Jahren noch weitreichende Parallelen auf wiesen, vgl. Rodney C. Hills, Predictaments in Polynesia: Culture and Constitutions in Western Samoa and Tonga, Pacific Studies 16 (1993), S. 115 ff.

49 Insb. Yash Gai / Jill Cottrell, Heads of State in the Pacific: a Legal and Constitutional Analysis, Suva 1990.

50 Diese spielten zuletzt in der fidschianischen Verfassungsreformdiskussion eine wesentliche Rolle.

51 Vgl. auch Peter Larmour, "A foreign flower"? Democracy in the South Pacific, Pacific Studies 17 (1994), S. 45 ff. 
Beziehungen, Verpflichtungen gegenüber Religionsgemeinschaft, Verwandschaft, Stamm oder der eigenen Insel (letzteres insb. etwa in Vanuatu ${ }^{52}$ ) hindern die Ausbildung stabiler politischer Konstellationen wie auch einer neutralen Beamtenschaft ${ }^{53}$. In Vanuatu entführte und inhaftierte das Militär Ende 1996 kurzerhand den Präsidenten Vize-Premierminister, im wesentlichen ging es um ausstehende Sold-Zahlungen ${ }^{54}$. Papua-Neuguinea stand im Frühjahr diesen Jahres aufgrund eines mit dem Bougainville-Konflikt zusammenhängenden Söldner-Skandals um seinen Premier-Ministers Julius Chan (der schließlich abtrat) am Rande eines gewaltsamen Umsturzes ${ }^{55}$.

Fidschi - geographisch-ethnisch Melanesien, politisch-traditionell eher Polynesien zugehörig - ist der südpazifische Staat mit der zur Zeit größten staatsorganisationsrechtlichen Dynamik $^{56}$. Gleich zwei Grundkonflikte belasten den nach Papua-Neuguinea größten Staat der Region. Einerseits ist der schon erwähnte Konflikt zwischen der indigenen und der "indischen" Bevölkerung ungelöst, andererseits ringt die Elite der "Chiefs" auch innerhalb der eigenen Ethnie um ihre Vormachtstellung. Schon die Unabhängigkeitsverfassung des Jahres 1970 trennte hinsichtlich des Wahlsystems zwischen den Ethnien, konnte freilich nicht den erwähnten "indischen" Wahlsieg des Jahres 1987 verhindern. Auch die traditionelle Privilegierung der Chiefs blieb nach der Unabhängigkeit erhalten, die schrittweise Erosion ihrer gesellschaftlichen Stellung blieb gleichwohl nicht aus. Es ist schwer zu beurteilen, welcher Konflikt den eigentlichen Ausschlag gab, jedenfalls eskalierte nach den Wahlen 1987 und der Ankündung eines Angriffs auf die Landpacht-Privilegien der Chiefs die Situation. Schließlich kam es zum Militärputsch; mit Waffengewalt - wenn auch unblutig - wurde die Regierung abgelöst und die Verfassung außer Kraft gesetzt. Auf der Grundlage der 1990 oktroyierten Verfassung, die den ethnischen Fidschianern eine deutliche Mehrheit im Parlament garantiert, wurde der Offizier, Laienpriester und Putschführer Rabuka 1992 zum Ministerpräsidenten gewählt. Dies ist er noch heute. Die Diskussion um eine Reform der Verfassung befindet sich in diesem Sommer 1997 in ihrer entscheidenden Phase. Auf der Grundlage des 1996 vorgelegten Berichts einer dreiköpfigen Verfassungsreformkommission ${ }^{57}$, der einen weitestgehenden Abbau der Inder-Diskriminierung vorschlug, hat sich ein "Joint Parliamentary Select Committee" nach schwierigen Verhandlungen auf ein Kompromißpaket geeinigt, das insbesondere die garantierte "fidschianische"

52

Den Brauch des "Wan-tok", der Vetternwirtschaft bei Ämtervergabe und Ämterkreation, beschreibt auch der "Annual Report 1995" der Ombudsfrau (Vanuatu), S. 3.

Isikili Mataitoga, Westminster Style Democracy and Cultural Diversity: A Critique of the Fijian Experience, in: Culture and Democracy in the South Pacific, Suva S. 90 ff. (zu Fidschi); Larmour (Anm. 11), S. 104 (zu Papua-Neuguinea).

Pacific Islands Monthly, Decembre 1996, S. 42.

Pacific Islands Monthly, May 1997, S. $12 \mathrm{ff}$.

Näher zu Verfassung und Recht in Fidschi Menzel (Anm. 2), passim.

Parliament of Fiji, Parliamentary Paper No. 34 of 1996, Textauszug in VRÜ 1997, S. 74 ff. 
Mehrheit im Parlament beseitigt. Dem Reformvorschlag hat - nachdem sich insbesondere Rabuka vehement für ihn eingesetzt hatte - schließlich auch die Zustimmung des traditionell konservativen Great Council of Chiefs gefunden ${ }^{58}$. Er dürfte nunmehr alle Aussichten auf Umsetzung haben. Wie ein Beobachter anmerkte, sind in Fidschi zur Zeit alle drei Typen legitimer Herrschaft nach Max Weber im Konflikt: die legale Herrschaft (der Verfassung) mit der traditionellen (des Great Council of Chiefs) und der charismatischen (des Putschführers Rabuka) 59 .

Ein einzigartiges staatsorganisationsrechtliches Gefüge findet sich in West-Samoa. Zwei der ranghöchsten Adeligen teilten sich seit der Unabhängigkeit 1962 das Amt des Staatsoberhauptes auf Lebenszeit; der eine starb im folgenden Jahr, der andere hat das Amt noch heute inne. Nach seinem Tod wird das Staatsoberhaupt auf jeweils fünf Jahre zu wählen sein. Das aktive wie das passive Wahlrecht zum Parlament blieb zunächst auf die matai (den Adelsstand) beschränkt. Dies ist zwar in der Verfassung nicht ausdrücklich vorgesehen, wurde aber durch ein allgemeines Referendum bestätigt. Der Titel des matai wurde in der Folge umso begehrter. Nun läßt die Tradition die Vermehrung des Titels zu - er reproduziert sich gleichermaßen selbst - und aus diversen Gründen, nicht zuletzt auch wegen des genannten Wahlrechtsprivilegs, "vermehrte" er sich erheblich. Der Streit um den Titel, schon immer bedeutsam, wurde zu einem der wichtigsten politischen Probleme in Samoa. Der von der deutschen Kolonialverwaltung eingerichtete Land- and Titles Court, der Streitigkeiten um Titelrechte abschließend klärt, gilt in Samoa wichtiger als das im übrigen höchste Gericht, der Court of Appeal. Eine Volksabstimmung hat 1990 das allgemeine aktive Wahlrecht gebracht. Wählbar sind nach wie vor lediglich matai. Der Court of Appeal des Landes hat 1995 die Einführung des allgemeinen aktiven Wahlrechts ebenso für verfassungskonform erklärt wie er früher die Beschränkung auf die matai für zulässig gehalten hatte. Gesetzgeberische "Systemwahlfreiheiten" im Wahlrecht sind offensichtlich ein weltumspannendes Phänomen. Die letzte - sehr umstrittene - Verfassungsänderung hat das samoanische Parlament im März diesen Jahres beschlossen. Nachdem der Rechnungsprüfer der Regierung in seinem 1994 vorgelegten Bericht massive Regierungskritik geübt hatte, wurde seine Amtszeit nunmehr kurzerhand auf je drei Jahre verkürzt ${ }^{60}$.

Nicht unerwähnt bleiben darf an dieser Stelle nochmals das Königreich Tonga. Wie erwähnt, die Monarchie ist vollsäftig, der König dominiert Regierung und Parlament gleichermaßen. Der traditionelle Adel wurde bereits im 19. Jahrhundert weitgehend entmachtet (insgesamt nur etwa 30 Titel-Träger blieben übrig), das Verwaltungssystem auf den König ausgerichtet. Die Königswürde ist erblich, gesichert ist die königliche Linie durch ein

Pacific Islands Monthly, July 1997, S. 7.

Larnour (Anm. 11), S. 101.

60

Samoa Observer v. 13. März 1997; zur Vorgeschichte Pacific Islands Monthly, February 1997, S. $25 \mathrm{f}$. 
verfassungsmäßiges (vom aktuellen Amtsinhaber schon mehrfach ausgeübtes) Veto-Recht gegen Heiratspläne von Familienangehörigen. Das Legislativ-Versammlung besteht aus den 12 vom König ernannten Angehörigen der Exekutiv-Spitze ${ }^{61}$, neun gewählten Adeligen und neun gewählten Volksvertretern. Dieses auf den König zentrierte System der Machtausübung ist seit einigen Jahren wachsendem Druck durch eine organisierte Pro-Demokratie-Bewegung ausgesetzt ${ }^{62}$. Der derzeitige - in der öffentlichen Meinung fast sakrosankt erscheinende - König freilich widersetzt sich beharrlich (und verweist dabei unter anderem auf die schlechten Erfahrungen, die Deutschland mit plötzlicher Demokratisierung in der Weimarer Republik gemacht habe). Schritte in Richtung einer Demokratisierung werden vor einem - angesichts seines fortgeschrittenen Alters vielleicht nicht fernliegenden Wechsel an der Spitze kaum erwartet.

\section{Grund- und Menschenrechte}

Die meisten der südpazifischen Staatsverfassungen haben umfangreiche Grundrechtskataloge. Zwar besteht im Grundsatz üblicherweise Justiziabilität ${ }^{63}$ (ohne daß es spezielle Verfassungsgerichte gäbe), oft sind aber schon die Grundrechtswährleistungen selbst mit weitreichenden Einschränkungsvorbehalten verbunden (in der Verfassung Fidschis sind etwa alle Ungleichbehandlungen von Fijians und Indians durch einschlägige Regelungen "abgesichert").

Gegenstand andauernder Diskussionen ist die Pressefreiheit. Einer Funktion der Medien als "vierter Gewalt" stehen dabei freilich zunächst weniger staatliche Restriktionen irgendwelcher Art im Wege, sondern schlicht in der Minimalität der Märkte. Nur Papua-Neuguinea und Fidschi verfügen unter den Kernstaaten des Südpazifiks über eine Tagespresse. In Tonga, West-Samoa, Vanuatu den Solomonen usw. finden sich allenfalls halb- bzw. wöchentliche Zeitungen. Kontrolle durch eine "vierte Gewalt" kann auf dieser Grundlage nur beschränkt stattfinden. Was die rechtlichen Beschränkungen der Presse anbelangt, variiert die Situation deutlich ${ }^{64}$. Fidschi verfügt zur Zeit (nach extremer Zensur in der Putsch-Phase 1987) über eine insgesamt recht freie und durchaus regierungskritische

61

62 gruppen Ha'apai und Vava'u.

Hierzu etwa I. C. Campbell, The Emergence of parliamentary politics in Tonga, in: Pacific Studies 15 (1992), S. 77 ff.; James Kerry, Tonga's pro democracy movement, in: Pacific Affairs 67:2 (1994), S. 242 ff.; Martin Tiffany, Tonga's call for democracy, Pacific Islands Monthly, Jan. 93, S. $16 \mathrm{ff}$.

63

64 
Presse. Die Diskussion um die Grenzen der Pressefreiheit lebte hier gelegentlich auf, etwa nachdem die Fiji-Times mit dem Foto eines im Parlament schlafenden Chiefs aufgemacht hatte, oder zuletzt, als die Regierung einem ansässigen Australier seine regierungskritische Kolumnistentätigkeit verbieten wollte (die derzeitige Verfassung verlangt ausdrücklich die Achtung der "Fijian values" durch die Presse ${ }^{65}$ ). In Tonga ist Kritik am Königshaus von der Garantie der Pressefreiheit ausgenommen ${ }^{66}$. Besonders problematisch ist die Situation etwa in Vanuatu ${ }^{67}$ : Über den journalistisch tätigen Ausländern schwebt hier fortwährend das Damokles-Schwert der - rechtlich nicht begründungsbedürftigen - Ausweisung (die Ausweisung ist, das sei hier nur angemerkt, in der Tat ein Grundproblem, das auch die Wirtschaft Vanuatus erheblich zu beeinträchtigen scheint ${ }^{68}$ ). In Papua-Neuguinea wird im Bürgerkriegsgebiet Bougainville die freie Berichterstattung seit Jahren weitestgehend unterbunden.

Der Südpazifik ist christlich und seit seiner Entdeckung bevorzugtes Aktionsfeld bisweilen recht fundamentalistischer Religionsgemeinschaften. Insbesondere im sog. "Bibel-Gürtel" von Fidschi über Tonga nach West-Samoa ist, unbeschadet der Tatsache, daß die Freiheit der Religion regelmäßig verbürgt ist, auch die Ordnung des öffentlichen Lebens an spezifisch christlichen Wertungen ausgerichtet. Dies gilt insbesondere für die Beschränkung aller öffentlichen Betätigung an Sonntagen, die etwas der Tourismus-Branche zu schaffen macht $^{69}$. In samoanischen Dörfern kommt es bis heute zur förmlichen Abstrafung der Verweigerung des Kirchgangs. Tokelau plant, außereheliche Geschlechtsbeziehungen unter Strafe zu stellen ${ }^{70}$. Vanuatu (ansonsten recht säkular) wies Ende 1996 unter Berufung auf christliche Werte den Lebensgefährten eines kanadischen Juristen aus, der gerade als Mitarbeiter der Ombudsfrau eingestellt worden war $^{71}$. Zu den vom Great Council of Chiefs im Zuge der fidschianischen Coups 1987 formulierten Forderungen gehörte es, "sicherzustellen, daß das Christentum die Religion für jedermann in Fidschi" sei ${ }^{72}$, das Christentum zur Staatsreligion zu erheben - bei etwa 50 \% Christen in der Bevölkerung. Zuletzt spielte die

65

66

67

68

69

In West-Samoa und Tonga resultiert hieraus gesellschaftspolitisch im übrigen wegen der christlichen Homogenität nur ein begrenztes Problem. Anders ist die Situation im religiös heterogenen Fidschi, wo im Zuge der Coups 1987 ebenfalls ein strenger (Ende 1995 wieder aufgehobener) Sunday-Ban verhängt wurde.

70

71

72

Constitution of Fiji, Section 19 (2) (d).

Section 7 der Verfassung Tongas von 1875.

Vgl. Pacific Islands Monthly, August 1996, S. 19 ff.

Hierzu Alan Ward, Rural Alienated Land in Vanuatu: Ten Years after Independence, in: Crocombe/Meleisea (Anm. 38), S. 104.

Vgl. A.H. Angelo, "Tokelau", in: Ntumy (Anm. 33), S. 307.

Vanuatu Trading Post vom 1. März 1997.

Zitiert nach Isikili Mataitoga, Constitution Making in Fiji: a search for a practical solution, in: Melanesian Law Journal Vol. 19 (1991), S. 43 ff. 
"Christianity" des fidschianischen Staates in der Schlußphase der Diskussion um die Verfassungsreform noch einmal eine erhebliche Rolle, der Great Council of Chiefs hat seine Zustimmung zum Verfassungsreform-Paket mit der erneuten Forderung verbunden, über die Erklärung Fidschis zum "Christian State" zu verhandeln ${ }^{73}$.

Ein allgemein bedeutsames Problem, das auch die Diskussion vor Ort beherrscht, ist das Spannungsverhältnis zwischen indigener Tradition und staatlicher Grundrechtsgewährleistung. Ein liberales Grundrechtskonzept läßt sich mit der traditionalen Gruppenordnung der üblicherweise hierarchisch-patriarchalischen Dorfgemeinschaft nur begrenzt harmonisieren. Dieser latente Konflikt wird durch die Vorbehalte zugunsten der Traditionen innerhalb der Grundrechtskataloge durchaus auch formal dokumentiert. Die derzeitige Verfassung Fidschis hält solche Klauseln (Respekt gegenüber den Chiefs usw.) in Fülle, in West-Samoa gilt das Verbot der Zwangsarbeit nicht für traditionelle Arbeitspflichten im Dorf ${ }^{74}$; die Verfassung der Solomonen erklärt das Diskriminierungsverbot hinsichtlich das Customary Law für unanwendbar ${ }^{75}$. Tuvalu stellt seine Grundrechte ganz generell unter den Vorbehalt, daß sie nur in Vereinbarkeit und Respekt für die "Tuvaluan values and culture" ausgeübt werden können ${ }^{76}$.

\section{E. Customary Law}

\section{Anerkennung als Rechtsquelle}

Die meisten Verfassungen der Region erkennen traditionelles Gewohnheitsrecht ausdrücklich an. Zuletzt haben etwa die Cook-Inseln ihre Verfassung dahingehend ergänzt ${ }^{77}$. Der zugesprochene Rang variiert, regelmäßig steht es unter der Verfassung und dem kodifizierten Gesetzesrecht. Das Verhältnis zum Common Law bleibt demgegenüber formal oft unklar, in Kiribati räumt die Rechtsprechung dem Gewohnheitsrecht insoweit Vorrang $e^{78}{ }^{78}$. Präzisierungen zur Anwendbarkeit von Gewohnheitsrecht finden sich im einfachen Recht, gelegentlich sogar im Rahmen spezieller Gesetzgebungsakte. In Papua-Neuguinea ${ }^{79}$

73

74

75

76

77

79
Pacific Islands Monthly, July 1997, S. 7.

Western Samoa Constitution, Part II, Art. 8 (d).

Solomon Islands Constitution, Section 15 (5)(d).

Tuvalu Constitution, Section 11 (2) (b).

Verfassungsänderung Cook Islands 30.06.1995 (Pacific Constitutions, Supplementary Readings, unveröffentlichter USP-Druck, Port Vila (Vanuatu) 1997.

Vgl. Martin Tsamenyi, "Kiribati", in: Ntumy' (Anm. 33), S. 78.

Auf den Solomenen ist eine entsprechende Regelung auf dem (wie zumeist in Melanesien langen) Weg; vgl. Jennifer Corrin, "Solomon Islands", in: Cheryl Saunders / Graham Hassall (ed.), AsiaPacific Constitutional Yearbook 1993, Melbourne 1995, S. 262 ff. 
existiert ein spezieller "Customs-Recognition-Act", in dem es um die Anwendungsfelder für Gewohnheitsrecht im Gerichtsverfahren geht, aber auch um Fragen der Beweisfindung. Das Gesetz anerkennt und limitiert die Berücksichtigung von Gewohnheitsrecht damit gleichermaßen. Seine Anwendung ist ausdrücklich ausgeschlossen, wenn dies ungerecht wäre, gegen das öffentliche Interesse oder das Wohl eines Kindes unter 16 Jahren ver stieße ${ }^{80}$.

Materiell ist das indigene Gewohnheitsrecht - nimmt man wieder die Region als ganze in den Blick - in erster Linie im Hinblick auf die Regeln der dörflichen Ordnung und des Zusammenlebens und Fragen des Familien- und Erbrechts bedeutsam. Im Bereich des Strafrechts kommt zum Teil den traditionellen Wiedergutmachungsmechanismen (etwa dem fidschianischen "bulubulu", der Überreichung eines Walfischzahns an das Oberhaupt der Familie oder des Dorfs des Opfers) strafersetzende oder strafmildernde Bedeutung zu. Im übrigen kann die Traditionsgemäßheit unter bestimmten Voraussetzungen auch die Einsichtsfähigkeit in das Unrecht (und damit die Strafbarkeit) entfallen lassen, wie dies etwa für kannibalische Praktiken im Hochland Papua-Neuguineas entschieden wurde ${ }^{81}$.

\section{Landrecht}

Das rechtspolitische Problem des Südpazifiks ist das Landrecht. Über keine Materie gibt es einen auch nur andeutungsweise vergleichsweisen Bestand an wissenschaftlicher Literatur, über keinen Bereich wird so flächendeckend gestritten und in keinem anderen Feld spielt das indigene Gewohnheitsrecht (oder auch das, was man dafür hält bzw. ausgibt) eine solche Rolle. Dabei unterscheidet sich die Situation in den Inselstaaten grundlegend von derjenigen in Australien und Neuseeland, wo die Landeigentumsrechte der Aborigines bzw. Maori zur Zeit ebenfalls die politische wie juristische Diskussion beherrschen. Anders als dort, wo an den gesellschaftlichen Rand gedrängte Ureinwohner um Rehabilitation kämpfen, ist die indigene Bevölkerung auf den unabhängigen Inseln regelmäßig "herrschend". Hier ist das traditionelle Landrecht oft schon verfassungsrechtlich gewährleistet und das Land vor der Veräußerung an Ausländer geschützt.

In Fidschi ist $82 \%$ des Landes "native land" und steht im Eigentum traditioneller Einheiten der indigen fidschianischen Bevölkerung (der mataqali). Der indische Teil der Bevölkerung ist auf das verbleibende "freehold-land" oder auf den Abschluß von Pachtverträgen verwiesen $(30 \%$ der Erlöse aus diesen Verträgen gehen unmittelbar an die Chiefs der verschiedenen Ebenen, $45 \%$ an den mataqali und $25 \%$ an die für die Verwaltung zuständige Native

80

81

Customs Recognition Act, Section 3 (1).

Regina v. Noboi-Bosai and others, Papua \& New Guinean Law Reports, 1971-72, S. 271 ff. 
Land Commission). Die dem System zugrunde liegende Eigentümerstellung beruht auf der in dieser Verallgemeinerung unzutreffenden Vorstellung der englischen Kolonialverwaltung vom traditionellen Recht in Fidschi ${ }^{82}$. Die Erfindung einer neuen Tradition war freilich erfolgreich, heute sind auch die meisten Fidschianer von ihrer Authentizität überzeugt.

In Vanuatu, wo insbesondere französische Siedler während der Kolonialzeit großflächig Eigentum erworben hatten ${ }^{83}$, erklärt die Unabhängigkeitsverfassung des Jahres 1980 alle solchermaßen erworbenen Eigentumstitel für unwirksam ${ }^{84}$. Soweit sie "ihr" Land behalten wollten, mußten die bisherigen Eigentümer nun Pachtverträge mit den "kastom-owners" abschließen (soweit diese sich dazu bereiterklärten; soweit nicht, blieb es bei einer Entschädigung investiv bewirkten Wertzuwachses, der Eigentumsverlust selbst wurde nicht entschädigt). Die Rebellion gegen den neuen Staat, die 1980 auf den Inseln Espiritu Santo und Tanna ausbrach, wurde nicht zuletzt von den betroffenen Siedlern getragen ${ }^{85}$. Gerade in Vanuatu wird das traditionelle Landrecht heute als Entwicklungshemmnis angesehen. Agrarexperten beklagen das Brachliegen wertvoller Anbauflächen aufgrund traditioneller Tabus und die investitionsverhindernde Unsicherheit der Pachtverträge. Problematisch ist aber vor allem die lähmende Wirkung, die von den immer wieder auch gewalttätig ausgetragenen Zwistigkeiten über die gewohnheitsrechtliche Zuordnung von Land ausgehen.

Die Unsicherheiten des Gewohnheits-Landrechts sind freilich ein allgemeines Problem der Region. Einige Staaten wie Fidschi oder Tuvalu haben hier durch Kodifizierung abzuhelfen versucht. Dies erhöht zwar die Rechtssicherheit, hat aber den großen Nachteil, daß die Systeme ihre natürliche Flexibilität (wie sie heute etwa noch auf den Cook-Inseln besteht) verlieren. Andere Staaten (wie West-Samoa mit seinem Land and Titles Court) sehen spezielle Entscheidungsinstanzen vor.

Nur angemerkt sei schließlich, daß es dem Landrecht parallele Fragestellungen zumeist auch zu Wasser gibt. Hier ist die Rechtssicherheit sogar noch geringer, und nicht nur in Fidschi und Vanuatu haben Fischerei- und Tourismusbranche mit erheblichen Problemen wegen unklarer und umstrittener traditionaler Nutzungsrechte zu kämpfen.

82

Josefata Kamikamica / Tim Davey, Trust on Trial - The Development of the Customary Land Trust Concept in Fiji, in: Yash H., Ghai (ed.), Law, Government and Politics in the Pacific Island States, Suva 1988, S. 284 f

Vgl. Weisbrot (Anm. 21), S. 69, nach dem die französischen im Vergleich zu den englischen Siedlern 1980 fünf mal mehr Land kontrollieren. Chapter $12 \mathrm{~d}$. Verfassung Vanuatus

Vgl. Alan Ward, Rural Alienated Land in Vanuatu: Ten Years after Independence, in: Land Issues in the Pacific, S. 97; Weisbrot (Anm. ), S. 72 f., der darauf hinweist, daß die Rebellenbewegungen ganz antagonistische Wurzeln hatte. 


\section{Customary Courts}

Ein wichtiges Thema der rechtspolitischen Diskussion in der Region sind die sogenannten Customary-Courts. Was aber unter diesem Begriff zusammengefaßt wird, hat bei näherer Betrachtung oft nur wenig miteinander zu tun. Bisweilen handelt es sich um kaum mehr als eine allgemeine - dem gerichtlichen Verfahren vorgeschaltete - Schlichtungsinstanz, gelegentlich um eine entformalisierte erste Gerichtsinstanz, vereinzelt auch um eine abschließende Gerichtsbarkeit für spezielle, dem Gewohnheitsrecht unterstehende Streitigkeiten. Pauschalierend läßt sich aber sagen, daß es in den Customary Courts regelmäßig um kleinere Angelegenheiten, solche des dörflichen Zusammenlebens und um die Wahrung von Tradition und Brauchtum geht. Das Verfahren ist im Vergleich zum "ordentlichen" Gerichtsverfahren mehr oder weniger konsequent vom strengen englischen Verfahrensrecht befreit, weist zum Teil insbesondere inquisitorische Züge auf. Es stellt in diesem Sinne oft eine Art Mischung aus aus Elementen traditionaler Streitschlichtung und moderner rechtsstaatlicher Gerichtsbarkeit dar. Um die Akzeptanz zu steigern, werden zum Teil die traditionellen Autoritäten als Entscheidungsinstanzen formalisiert. Anwaltliche Vertretung ist oft untersagt, um Schlichtung zu erleichtern.

Papua-Neuguinea gehörte 1975 zu den ersten Staaten, die "village courts" einrichteten. Der von der örtlichen Bevölkerung benannte Laienrichter kann hier Geldstrafen und bis zu sechsmonatige Gemeinschaftsarbeit verhängen. Er ist zuständig in Straf- wie Zivilsachen wie auch in Fragen der Einhaltung dörflichen Ordnungsrechts. In Fidschi ist die Wiedereinführung der (in den 60er Jahren praktisch stillgelegten) "Fijian Courts" Gebot der aktuellen (oktroyierten) Verfassung. Über das ob und wie der Umsetzung ist Jahre gestritten worden. Nachdem 1994 bereits die ersten Laienrichter eingestellt worden waren, ist die Umsetzung der Bestimmung nun wieder ins Stocken geraten. Der 1996 vorgelegte Bericht der Verfassungsreformkommission hat nun vorgeschlagen, auf spezielle Gerichte zu verzichten und statt dessen das ordentliche Gerichtsverfahren - etwa im Hinblick auf die Gerichtssprache - zu modifizieren.

Besonders problematisch ist der west-samoanische "Village-fono-Act" ${ }^{86}$. Hier wird die Bestrafungskompetenz "according to custom" anerkannt, ohne daß die inhaltlichen Grenzen klar aufgezeigt würden. Das Gesetz beschränkt die Gewalt des village fono nur personal auf die Dorfbewohner und ordnet die Justiziabilität seiner Entscheidungen an. Hinsichtlich der zulässigen Strafmaßnahmen wird auf die traditionellen Strafen in Geld, Nahrungsmitteln und Matten verwiesen, ohne daß diese Aufzählung aber als abschließend formuliert wäre. So ist etwa die Frage, ob dem village fono das Recht der Verbannung von Dorfbewohnern 
zukommt, ungeklärt - der Court of Appeal hat sie kürzlich ausdrücklich offen gehalten ${ }^{87}$. Im Zusammenhang der bereits erwähnten Tötungsanordnung in Lona-Village scheint unter den matai zunächst sogar die Auffassung verbreitet gewesen zu sein, daß der "village fono act" auch solches rechtfertige ${ }^{88}$. So abwegig dies unter der Verfassung ist, es zeigt die Sprengkraft derartig unklarer Regelungen (ein Parlamentsmitglied soll im Zusammenhang des Loma-Falls die Verhaftung des Premierministers verlangt haben, der verantwortlich für den village fono act gewesen sei und damit auch für die Tötung ${ }^{89}$ ).

\section{F. Schluß}

Der Südpazifik erweist sich nicht nur ethnisch, sondern auch verfassungsrechtlich als geteilter Raum. Die Probleme der polynesischen Staaten Tonga und West-Samoa sind mit denjenigen der melanesischen Staaten Papua-Neuguinea, den Solomonen und Vanuatu kaum zu vergleichen; Fidschi ist in mancher Hinsicht ohnehin ein Sonderfall. Bemerkenswert ist, daß die polynesischen und mikronesischen Gebiete, die schon bei Ankunft der ersten Europäer eine gewisse politische Struktur zeigten, heute erheblich stabiler sind als die als einheitliche Herrschaftsgebiete völlig neu entstandenen melanesischen Räume. In der gesamten Region vermischen sich Elemente moderner westlicher Demokratie mit formellen oder auch informellen Strukturen traditionaler Gesellschaft. Der politische Prozeß wie auch der Rechtsalltag sind von diesen Strukturen auch dort bestimmt, wo sich dies in den Verfassungstexten nicht widerspiegelt.

Gemeinsam ist, daß die Berufung auf Tradition und Gewohnheit im Südpazifik einen großen politischen Wert hat. Der "pacific way", vom damaligen fidschianischen Präsidenten Ratu Kamasese Mara 1970 vor den Vereinten Nationen zum Programm erhoben, hat sich auch in den Rechtsordnungen und der Rechtspraxis niedergeschlagen. Manches, was im Südpazifik als Tradition beschworen wird, ist dabei nach dem Europäer-Kontakt und gerade in seiner Folge entstanden (was freilich nicht ausschließt, sie inzwischen als "traditional", jedenfalls aber als "customary" zu bezeichnen). 200 Jahre sind auch im übrigen an der Südsee nicht spurlos vorübergegangen. Dort, wo ein intensiver Kontakt mit der Außenwelt stattfindet, führt dies eben auch zur Etablierung neuer Werte. Traditionelle Dorfstrukturen und Hierarchien sind aber eben nicht mehr überall allgemeines Ideal ${ }^{90}$. Nur

Italia Taamale vs. Attorney General, C. A. 2/95B, v. 18.08.1995 - dem Land and Titles Court wird eine solche Anordnung freilich zugestanden.

88

89

Vgl. Lawson (Anm. 45), S. 156 f.

Lawson (Anm. 45), S. 157.

90 Sie ist hier eben auch zu sehen unter dem Gesichtspunkt, daß Chiefs, deren prozentualer Anteil an den Landpachtverträgen in den vergangenen 50 Jahren verdreifacht hat (wobei auch noch die kor- 
hingewiesen sei darauf, daß West-Samoa - einerseits besonders traditionell, andererseits in voller Kenntnis der (neuseeländischen) Gegenwelt - mit seinen noch weithin realen dörflichen Zwängen eine der höchsten Suizidraten unter Jugendlichen weltweit aufweist und daß in Fidschi der Widerstand gegen die Einführung der Customary Courts insbesondere von (gut organisierten) Frauengruppen ausgeht ${ }^{91}$.

Das Spannungsverhältnis ist also immer wieder augenscheinlich. Eindrucksvoll ist, in welcher demokratischen Offenheit in der Region diskutiert wird, wie der Weg zu finden sei zwischen "moderner" Verfassungsstaatlichkeit und der eigenen Kultur: eben der pacific way. Die im Weltvergleich fast "zivilen" (afrikanische oder südamerikanische Putschisten würden vielleicht sagen "schwächlichen") Militärcoups in Fidschi und die Entwicklung seither sind auch insoweit bemerkenswert.

relierenden Leistungspflichten weitgehend entfallen sind), um ihre Pfründe kämpfen; Crocombe, in: Crocombe/Meleisea (Anm. 38), S. $12 \mathrm{f}$.

Die Tätigkeit der village courts in Papua Neuguinea ist gerade im Hinblick auf die Wahrung von Frauenrechten auf Kritik gestoßen (vgl. etwa Owen Jessup, Customary Family Law, Women's Rights and Village Courts in PNG, Melanesian Law Journal 19 (1991), S. 65 ff.). 
Stockholm to Rio, both on a global level and regarding Africa, Asia and Latin America. In a larger sense, the Biodiversity Convention is also designed to put an end to the piecemeal approach which characterized nature conservation by international law in the past decades, not by substituting the other conventions, but by adding missing links and a common background. The concept of sustainable development as embodied in the Convention on Biological Diversity is such a link addressing the conflict between environment and development: It includes the concept of protection of species and their "variety" for future generations (intergenerational equity) within and outside of ecosystems and natural habitats, the duty to sustainable use natural resources, the concept of transfer of technology and equitable sharing of scientific knowledge and financial burdens, and the integration of biodiversity conservation schemes into national plans, programs and projects. Thus the concept of sustainable development, in the context of international environmental law on nature conservation, is far more than the concept of sustainable use which is part of it.

\section{Constitutional Law and Traditional Social Order in Southern Pacific Micro-States}

\section{By Jörg Menzel}

The South Pacific Region boasts more constitutional "per capita" than any other region in the world today. Fourteen states and a number of dependent territories are inhabited by only a few million people. The diversity of current constitutional structures reflect the history of the region. In general, "pre-contact-societies" in Melanesia were small and quite egalitarian whereas those of Micronesia and especially those of Polynesia tended to be highly stratified proto-states. With the arrival of Europeans and (subsequent) colonialisation, European legal systems were implemented throughout the region. This does not mean, however, that the legal traditions of the indigenous peoples have disappeared. Particularly in "indirect-rule"-systems as Fiji and Western Samoa legal traditions were to a certain extent accepted. Colonial authorities even confirmed the status of indigenous authorities on some occasions. These authorities (usually called "chiefs") also play an important role within in the current political systems. This is not only the fact in the monarchy of Tonga or in aristocratic Western Samoa, but also in Fiji, Vanuatu and elsewhere. Since decolonialisation attempts have also been made to strengthen the role of indigenous customary law within the legal system of the region. Customary law is recognized as a source of law within many constitutions, it is absolutely predominant within the area of land tenure. The tension between traditional rule and village life on the one hand, and the modern idea of democracy and individual human rights on the other make "the politics of tradition" particularly controversial, not only in highly stratified societies like Western Samoa, Tonga, and Fiji, but in the region as a whole. 